\title{
THE POSSIBILITY OF ELECTROMYOGRAPHY MEASURING AS THE ANSWER TO BREATH HOLDING
}

\author{
Mirko Ostojić, \\ Milan Milosavljević ${ }^{*}$
}

Singidunum University, Belgrade, Serbia

\section{Correspondence:}

Milan Milosavljević

e-mail:

mmilosavljevic@singidunum.ac.rs

\begin{abstract}
:
There is an inherent connection between nervous system and muscle activity during breathing process. Both muscle fiber activation and the propagation of nerve impulses are electrical in its nature, whilst many cognitive functions could be measured by EEG signals. So the logical assumption might be that different breathing patterns, initially spotted by the minute volume changing, could be seen by change in electrophysiology of muscle activity - electromyogram (EMG). This pioneer multidisciplinary investigation has been concentrated on the most passive breathing activity, the prolonged breath holding. The safe and comfort method of physiological breath holding method has been applied. Having in mind number of anatomic and physiological characteristics of human body the two regions for non-invasive EMG measuring had been proposed- the neck and the region of solar plexus. The first test was done by 30 healthy volunteers, the students of sport science. The clear electrophysiology activity in these two regions has been proved. The measured respirational, ECG and two EMG signals has been shown that the sample rate of $200 \mathrm{~Hz}$ is quite enough. This opens the new possibilities for further experiments to be performed by enlarged number of electropoligraphical signals simultaneously taken. Whilst approaching to the point of physiological answer of the breath holding, the concerning increase of both average power and power spectral density had been spotted, especially in the regions of delta and theta.
\end{abstract}

Keywords:

electrocardiogram, respirational signal, the muscle activity, average power, the power spectral density.

\section{INTRODUCTION}

The main respirational center of the human body is situated in $\mathrm{Me}$ dulla oblongata [1] where four functional parts could be discerned (Fig. $1)$. For our purposes the two of them are more important for our study. The first part is called Pneumotaxic center (PTC). It can be spotted at the top of Fig. 1 and it controls both the depth and the frequency of breathing. The direct result of its effect is measured clinically by the minute ventilation $(M V)^{1}$. The second part is the Ventral breathing center and it mainly controls the inspirium. The common characteristic for both of

1 the product of number of inspirium/expirium per minute, as well as the breathing depth. 
these regions is that they are most sensitive ${ }^{2}$ to the afflux of carbon-dioxide concentration [2], $\mathrm{PCO}_{2}$, inducing enlarge of minute ventilation afterwards. This influence could be seen both by the bigger depth of every breath as well as the enlarged number of breaths per minute. The enlarged product of breathing volume and the number of breaths per minute is always reaction to the higher value of the $\mathrm{PCO}_{2}$.

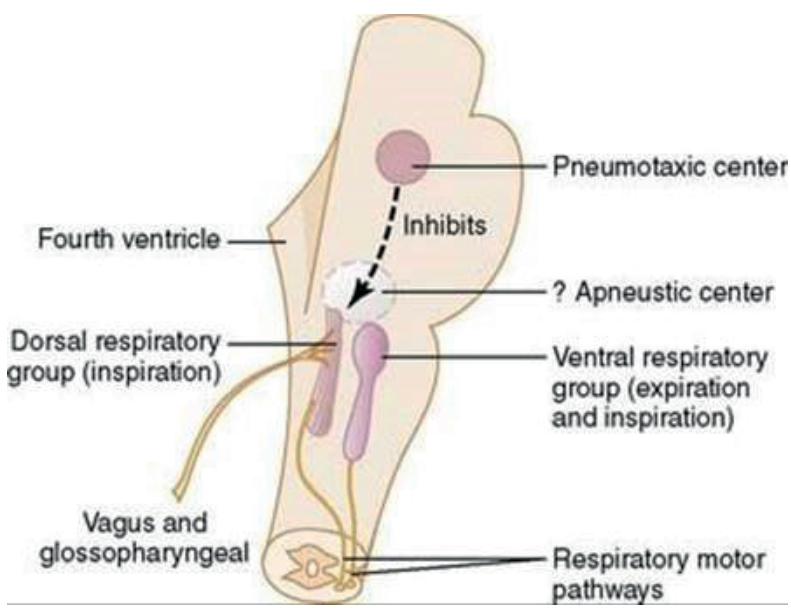

Fig. 1. Respirational centres

The innervation of certain muscle groups is of electrical type, while the true movement nature of myofibrils is contractile [3], what practically means that every single muscle perform action by its contraction. As the answer to the constant influx of $P C O_{2}, P T C$ sends more and more electrical impulses in fixed time frame inducing the increase electrical density in a muscle region, so the following sequence of events could be spotted [4]:

1. The breath holding induces the linear increase of $\mathrm{PCO}_{2}$.

2. PTC reacts to the increase of $\mathrm{PCO}_{2}$ by the increase of efferent ${ }^{3}$ neurons triggering frequency.

3. The different regions of respirational muscles have been aroused what would induce the process of inspirium during normal, spontaneous breathing.

2 The influence of the oxygen concentration on these centres is about 10 times less [1]. Also there are other gases involved, but all their influence is of minor importance to the influence of the concentration of carbon-dioxide.

3 also called motor neurons - are the nerves responsible for carrying signals away from the central nervous system in order to initiate an action.
4. After certain period, the triggering neuron frequency is so enlarged that the subject feels on the 'bump' sensation either in neck or in solar plexus region. This sense requires some practice to be properly recognized. In this phase, it is still possible to maintain willful holding of breath.

5. At certain moment, the innervation series of impulses is so dense that willful holding of breath becomes impossible, leading to a forced inspirium. ${ }^{4}$ Ganong labels this moment as the cutoff point [4]. The difference between cutoff point and the 'bump' time is non-linear in its nature and the only connection is the subject's ability to hold the breath.

The primal aim of this work has been the identification of possibility of measuring the electromyogram $(E M G)$ changes on certain muscle regions during certain variability of the breathing pattern. In order to even touch this problem, it was necessary beforehand to prove the insight of continues, measurable electrical activity [5] between two points on the outer skin surface.

If the answer to the above question would be positive, the second step might be the investigation of the general $E M G$ dynamics. This includes not only the changes in average power of consecutive signals, but also their frequency range. The latter result would give the answer to the necessary sample rate frequency. With lower frequency it would be possible to measure more signals simultaneously on the same equipment. Practically this would give the answer the question if we can even tackle measuring full EEG-cap at the same moment with measuring $E M G$ and $E C G$ signals.

The current clinical standard of measuring $E M G$ is slightly invasive method, inserting electrode needles to the both ends of a locomotor muscle [6,7]. It shows the spectral dynamics of maximum $450 \mathrm{~Hz}[8,9]$. If we include the muscle activation during the acquisition period, this process becomes even more complex. Since we had wanted to include only surface, non-invasive, skin electrodes, the frequency dynamics would be the last question to this research.

\section{PROCEDURE}

The thirty young, healthy students of National faculty of sport were beforehand learnt to the procedure of breath holding after spontaneous exhalation, as well to

$4 \quad$ this theoretical maximum period of breath holding is called maximal pause, MP. 
the recognition of physiological muscle activity answer as to a prolonged breath holding. ${ }^{5}$ The typical setup is shown at Fig. 2.

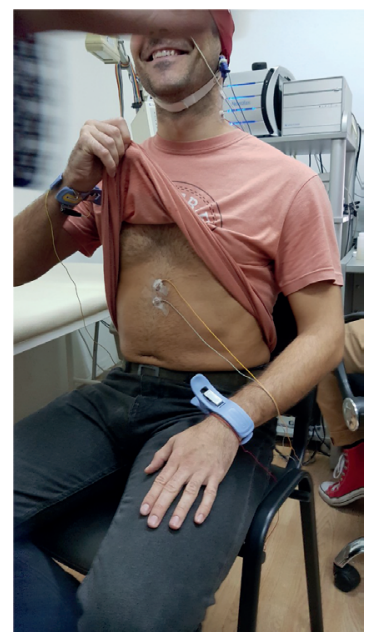

Fig. 2. Electrode setup at Neurofax equipment.

In order to perform the data acquisition for our purposes we have had the Japanese apparatus Kohden's Neurofax including very professional, educated personnel. This multichannel equipment supports measuring a large scale of electropoligraphical signals:

- the complete EEG cap, with sixteen channels and two pairs of grounding electrodes,

- ECG according to Einthoven,

- respirational signal on the nose tip, with simple task to measure the breathing airflow.

- largest palette of bipolar EMG electrodes, whilst we used the best possible surface $A u$-electrodes.

- HD-camera built-in to this system so the complete process of data acquisition had been simultaneously recorded.

- light hand built-in switch, for the event labeling - it was hold in subject's left hand, so he could mark the events of interest.

After the successful data acquisition, for the purposes of this study we used the four signals out (Fig. 3): ECG, $E M G$ signals of neck and solar plexus regions, as well one RESP signal on the nose tip as the airflow controller.

The neck signal had been measured at the ends of Sternocleidomastoids muscle ${ }^{6}$, while two points of solar

5 The given time is labeled on West as $\mathbf{C P}$ - control pause, while Russian term is контрольная пауза (КП)

6 this strong neck muscles has also the important role during the inspirium [1]. plexus region were situated on sternum's right side at the beginning and end of first sub rib arch. We wished to reduce the influence of the heart muscle wherever we could, so that is the reason why we measured the right side of the sub rib arch.

The whole recording procedure is non-invasive, lasts less than five minutes and Neurofax equipment corroborates the ethical standards of Serbian Medical Society.

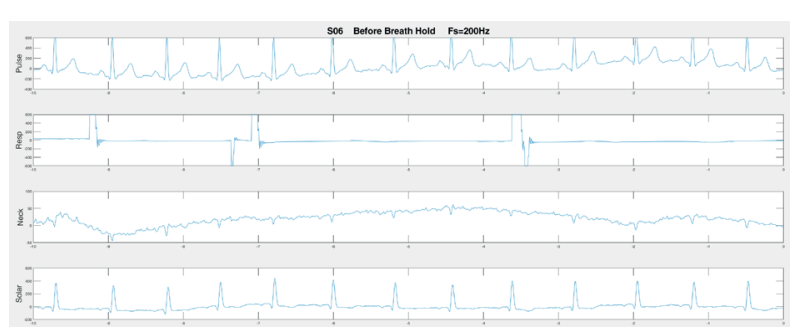

Fig. 3. The time domain of the recorded signals.

\section{RESULTS}

After the recording of 30 volunteers we got 30 raw time signals. After removing the artefacts, we extracted four groups of signals, according to the natural time sequence:

- natural state, without any activity, before the commencing of breath holding;

- the phase just after the beginning of breath holding;

- the state before the physiological answer to prolonged breath holding, and we call it before 'bump';

- the last, continual phase just after 'bump' with the relaxation period.

Wherever it was possible we obtained up to $10 \mathrm{sec}-$ onds of continuous signal absent of any artefacts. The heart artefacts weren't treated and that will be explained latter in this work.

At this point we got thirty groups of four signals: normal state (before breath hold), phase after commencing the experiment, the last phase before the 'bump', and the relaxing state after the 'bump'. It was total of 120 signals. Each of them is labeled in form $S_{x y}$ with corresponding phase.

Full EEG-cap and the RESP recorded signals are out of the scope of this work.

Fig. 3 clearly shows ECG, RESP and two EMG signals of the neck and solar region. The typical, healthy 
behavior of ECG can be noted, while signal RESP accurately indicates the air flow during both inspirium and expirium.

At the first outlook at $E M G$ signals it is easy to spot the influence of $E C G$, especially in the solar region since the proximity of heart muscle. This influence had been minimized by the slightly original method of signal analysis [10].

The first analysis we performed was the change of signal's average power in the time domain (Fig. 4). Neurofax equipment records the signals in $\mu \mathrm{V}$ of every single sample on the unit resistance. The current power can be get in $\mu \mathrm{W}$ by simple powering this value [11].

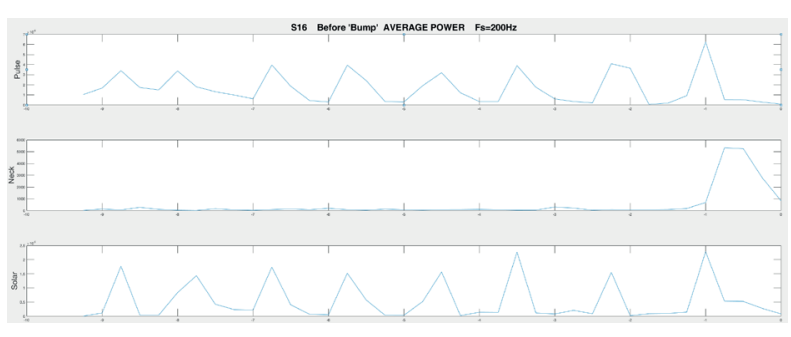

Fig. 4. The signal's average power in time.

In order to get smooth line of these changes we had used the well-known procedure of overlapping regions of digital signals analysis [12]. The correspondent programs for analysis has been attached with all given concrete values. Here we emphasize that the signal width is equal of $2 s$ since the main disruptive signal is $E C G$. In the case of sample frequency of $200 \mathrm{~Hz}$, the concrete value of 400 samples had been taken, with overlapping region of 50 samples. In the case of sample rate of $2 \mathrm{kHz}$ the proportional 10 times values have been adjusted.

The serious influx of power has been spotted just before the 'bump' in all cases, mainly in neck region, but not exclusively! Fig. 5 shows the period just before the 'bump' at the volunteer number 24 . We can spot the reaction in solar plexus region, and practically no reaction in the neck region. The signal propagation of signal till conscious reactions corroborates modern researches and it goes from $0.2 s[13]$ till $1.42 s[14]^{7}$.

$7 \quad$ The researchers include different cumulative periods. The full propagation may include the sum of the following steps: time of the sense reaction to the stimulus (visual, auditory, sensual), signal propagation from the sense cells to the brain, time of the reaction of cortex, and the time of pulse propagation from cortex by motor neuron.

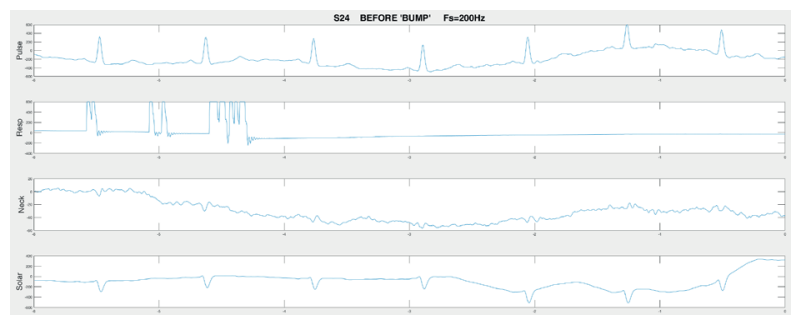

Fig. 5. The reaction in solar plexus region.

The most of the results show the reaction in the neck region versus solar one, but the power afflux was from two to even seven times in corresponding areas without any exception.

We have already mentioned that the breath holding is possible to prolong even certain time after the physiological answer [15], what was expected to happen during our experiment. We may spot at Fig. 6 a typical case where the volunteer was holding his breath additional four seconds after the event. Later, all such cases had been verbally confirmed, commenting the lack of trust in their own feelings ${ }^{8}$. Fig. 6 clearly shows the reactions in both regions. The effect happened in solar regions approximately $4 s$ before the event, and the neck region reaction is about $2 s$.

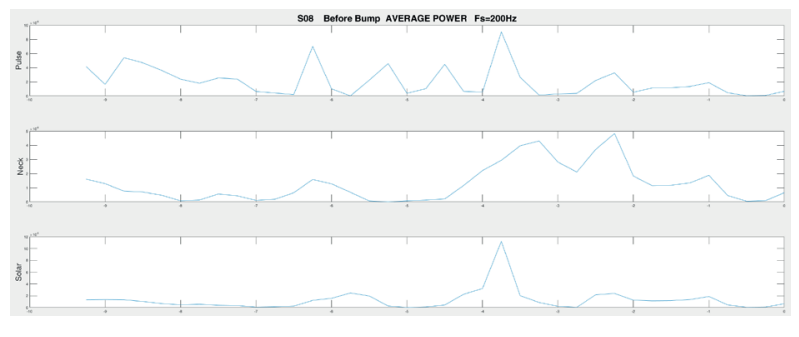

Fig. 6. Prolonged breath holding after the time of physiological answer.

After the positive answer to the first two question of our research, the possibility of getting the quality signals and the serious notice of average power changing of $E M G$ signals we commenced the spectral analysis without filtering low frequency components. The reason for this procedure was to spot the possible changes, not the exact values.

Computing the power spectral density [16] of EMG signals it had been determined that the given spectra

$8 \quad$ Here the sport spirit of our volunteers is inevitable. Sport is designed in its core for pushing forward personal limits. 
visibly doesn't exceed the value of $70 \mathrm{~Hz}$. From the practical point of view, the important conclusion is that over of $95 \%$ power spectrum power is situated in the region 0-40 Hz. This fact proves that the sample rate of $200 \mathrm{~Hz}$ is quite enough for this type of research, according to Nyquist [17].

The first insight at Fig. 7 shows the well-known, strong low frequency component that must originate from the heart activity (signals up to $1 \mathrm{~Hz} U L F$ spectrum). In this region can be spotted regularly high dynamics, while certain signals show also the distinct dynamism in the region of about $13 \mathrm{~Hz}$, corresponding to the center of standard alpha wave range.

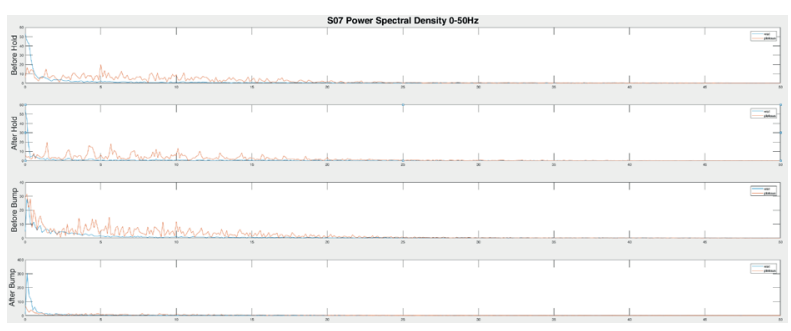

Fig. 7. The power spectral density of the both $E M G$ signals of typical volunteer. Blue lines depicts solar region, while red lines the neck area. The four periods are situated from the top to down. It is clearly visible the influx of power of neck signal in the phase of physiological answer. It can be also spotted that the fourth phase equalize its state as it was before the breath holding.

While observing the lower frequency at the Fig. 7, and especially the ULF part of the spectrum the few characteristic can be underlined:

1. Before the breath holding and at the commencing the procedure the $P S D$ in solar region is almost one order of magnitude above than the corresponding one in the neck area.

2. During the propagation of the experiment that ratio has been significantly reduced.

3. The neck area has the PSD afflux during the 'bump' and in most cases it becomes even higher than the temporary value of the solar region. This effect was present at all volunteers, regardless the mentioned results of the average power.

4. At the end of experiment, we have the exact picture as of the beginning of experiment - the values have been set up to their initial values promptly, without any exceptions.

\section{CONCLUSION}

Our study confirms the possibility of non-invasive measuring EMG signals in the precisely defined solar and neck region without any ambiguities. During the lack of breathing activity, the dynamic of these signals is very similar to $E E G$, also of non-invasive type, so the frequency does not exceed $100 \mathrm{~Hz}$. It is necessary to underline that the surface, skin electrodes were applied. It is bold assumption that this must lead to the loss of some spectral components.

Completely all volunteers show the significant afflux of mean power value just before the physiological answer to breath holding. This value is of two to seven times higher than in the normal state. The additional peculiarity of this research shows that five of total number of 30 volunteers shows higher values in solar plexus region, so $16,67 \%$, while the neck region may remain total intact, showing no any changes. This is why we suggest measuring both of the regions simultaneously in future researches of this kind.

The significant changes of $P S D$ dynamics could be noticed during the progress of breath holding, and afterwards setting to their initial values quickly with the normalization of breathing pattern. This dynamism is most obvious in the region delta and theta, as well around the exact frequency of $13 \mathrm{~Hz}$.

This robust multidisciplinary study presented the results that different biological phenomenon exports to the region of modern electrotechnics, so opening the new possibilities for the series of new experiments in order to establish the standards for electrical constants respirational signals, instead of only existing mechanical and chemical data of breathing parameters. This transition includes not only non-invasive results acquisition, but enables the full space freedom of the human body research, in vivo, measuring the current breathing parameters in the scale from severely sick patients to the top sportsmen [5].

\section{REFERENCES}

[1] A.C. Guyton, Physiology of the human body, Sunder College Publishing, Philadelphia, 2005.

[2] Y. Henderson, "Carbon dioxide", Cyclopedia of Medicine, ed. by HH Young, Philadelphia, F.A. Davis, 1940.

[3] D. Rakovic, Biophysics, 2008, IASC \& IEFPG, Belgrade, 2008, pp. 132-149. 
[4] E.B. Kim, Ganong's Review of Medical Physiology, 24th Ed., McGraw-Hill, 2012.

[5] M. Ostojic, Breathing Skills, Pesic \& Sinovi, Belgrade, 2018.

[6] J.H.T. Vitasalo and P.V. Komi, Signal characteristics of EMG during fatigue. European Journal of Applied Physiology 37, pp. 111-121.

[7] V.A. Boxtel, Optimal signal bandwidth for the recording of surface EMG activity of facial, jaw, oral, and neck muscles. Psychophysiology, pp. 38, 22-34, 2001.

[8] R. Merletti and D.P. Torino, Standards for reporting EMG data. Journal of Electromyography and Kinesiology, 1999.

[9] D.C.J. Luca, The use of surface electromyography in biomechanics. Journal of applied biomechanics, 1997.

[10] M. Milosavljevic, Robust Digital Processing of Speech Signals, Springer, New York, 2017.
[11] (https://www.medwrench.com/equipment/4722/ nihon-kohden-eeg-1200.pdf)

[12] W.E. Sabin, Discrete-Signal Analysis and Design, Wiley 2007 New York.

[13] B. Libet, Time of Conscious Intention to Act in Relation to Onset of Cerebral Activity, Brain 106: pp. 623-642, 1983.

[14] M. Masao and M. Hallett, The timing of the conscious intention to move, Eur J Neurosci. Dec; 28(11), pp. 2344-2351, 2008.

[15] A. Rakimov, Normal Breathing, Amazon Digital Services, Toronto, 2014.

[16] J. Fourier, Théorie analytique de la chaleur, IRPHE, Sceaux, 1988.

[17] H. Nyquist, Certain topics in telegraph transmission theory, Trans. AIEE, vol. 47, pp. 617-644, New York, 1928. 